\title{
Mechanism of Clustering Transportation for Projectiles Mixed Storage in Magazines
}

\author{
Zhang Jianbin ${ }^{1,}$, , Ge Yang ${ }^{2,}$, Chen Yiyuan², and Gao Dengfeng ${ }^{2}$ \\ ${ }^{1}$ Northwest Institute of Mechanical and Electrical Engineering, Xianyang 712099, China \\ ${ }^{2}$ Mechanical and Electrical Engineering College, Harbin Engineering Uni, Harbin 150001, China \\ ageyang@hrbeu.edu.cn
}

Keywords: conflict; Petri net; decision model; mixed storage; CTP; load suitability

\begin{abstract}
To focus on the problems of low transport efficiency, poor ship-borne suitability of the present large and medium caliber naval guns, utilizing information correlation and integration technology, this paper put forward and built the decision-making method of intelligent transporting and supplying of projectiles in Petri net. The method used the technologies such as mixed storage, intelligent decision and real-time transport to actualize clustering transportation for projectiles (CTP) of magazines. Through the systematic research under the multiple working conditions such as start and stop and multi field coupling conditions of wind, wave and current. The result shows that: compared with conventional projectile transportation methods, the cycle and frequency of random transportation and replenishment of projectiles improve significantly with clustering transportation for projectiles of magazines in Petri net, which means the replenishment efficiency of naval gun is improved effectively, and the conflict problem in projectiles transportation and replenishment has been solved. The projectile transportation platform with CTP method realized not only the variable-speed transportation and replenishment of projectiles with the condition of compatible projectile transportation and replenishment, but also continuous efficient transportation and replenish of projectiles. Its volumetric rate and ship-borne suitability were improved obviously.
\end{abstract}

\section{Introduction}

There are prominent problems of low transport efficiency, poor ship-borne suitability on present large and medium caliber naval guns. Because of the difference between modern naval vessels' combat tasks and operational targets, different operational weapons require different projectile magazines. Therefore, similar ammunition platform storage, hybrid transport can greatly improve the warehouse system's shipborne fitness and ammunition supply efficiency, at the same time, bring a series of effects such as reducing shipborne equipment and energy consumption. However, in the continuous process of projectile transportation, simultaneously, transportation conflicts of projectiles are constantly formed, which directly leads to low efficiency of the transportation system. Meanwhile, 
the large number of projectiles and limited space of the naval vessel caused the space constraints. Constrained by low transportation efficiency and poor ship-borne suitability, the projectile transportation system should not only have the capability of automatic rapid transportation and replenishment of projectiles, but also have the capability of multi-task judgment and decision-making with complex and extreme conditions ${ }^{[1 \sim 2]}$.

A multi-level mechanism of projectile transportation platform is set up for a certain type of ship, and study the projectiles integration of transportation, return, replenishment, replacement and transportation and adopted different decision strategies to solve conflicts according to different specific tasks and objectives. The specific analysis methods mainly include artificial neural network analysis, correlation matrix method and clustering analysis and so on. The paper combined the intelligent transfer technology of naval gun projectiles, effectively solved the problem of projectile transportation conflict in naval gun projectiles transfer.

\section{Technical Proposal of Integrated Transportation}

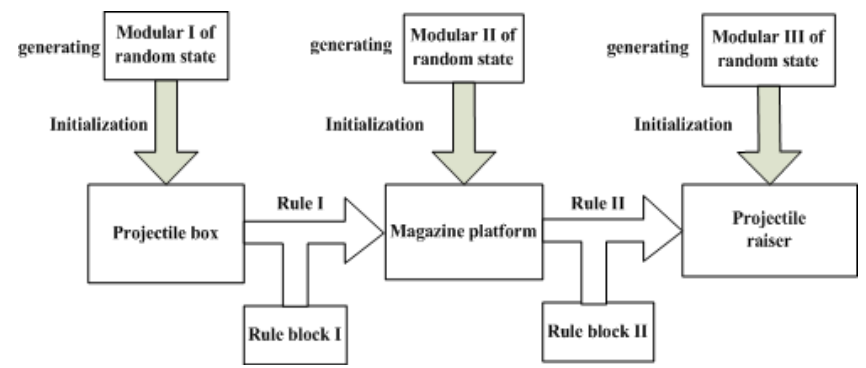

Fig. 1 Topology relation of integrated transportation system

As shown in Fig. 1, a large projectile transportation platform has a variety of projectiles. The projectile transportation process was completed by projectiles storage tank, projectile transportation platform and projectile hoisting mechanism. The new projectile transportation system consisted of the projectile mechanism including raisers, boxes and magazines for transferring, transportation and replenishment. The location of the projectiles was determined by the relative position of the projectile transportation device in the space at a certain time, which the projectiles at different positions is supplied and transported to the barrel by a certain order. The transfer of the projectile from the starting point to the terminal point is completed step by step by the various agencies, each working process is controlled by switch and digital signal, the number of projectiles in the magazine is random, and the type of projectiles is fixed. Intelligent projectiles transfer system used embedded planner and loader. Loader, planner and simulation display platform were embedded into the whole projectile magazines system. Horizontal axis is projectiles position, vertical axis is 1-A projectiles, 2-B projectiles, 3-C projectiles, using RFID radio frequency launching system to identify and detect the type of projectiles.

\section{Mechanism of Clustering Transportation for Projectiles}

Cluster analysis is a kind of classification and group technology, according to the order diversity and process complexity of projectile transportation system, the optimal sequence is selected and the projectile transportation process is designed reasonably. The logic of projectile transportation is obtained by cluster analysis in order to keep the working performance balance of system mechanism under dynamic load. 


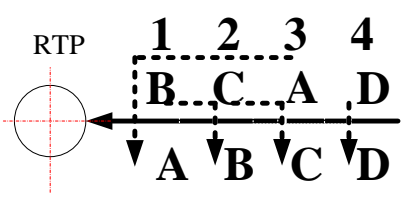

Fig. 2 Mechanism bullet vs. ammunition label

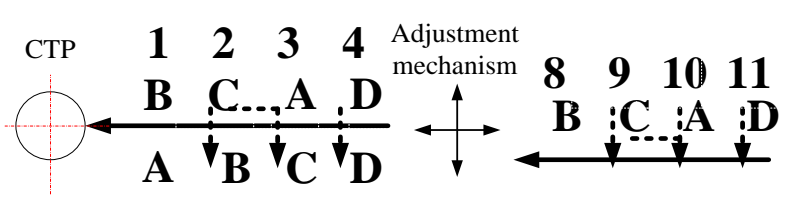

Fig. 3 CTP vs. RTP

Under the condition of continuous ammunition supply, time series analysis is the precondition of cluster analysis in order to improve the efficiency and shorten the ammunition replacement. As shown in Fig.2, routine tasks were decomposed into several separate sub-tasks according to the requirements of the task. Different sub-tasks were performed by different agencies, including: 1-4 for the mechanism bullet; A-D for the ammunition label; arrows represent the relationship between ammunition and projectile position adjustment.

As shown in Fig. 3, the work of the system under dynamic load was in dynamic equilibrium. Taking n-successive launches as an example, when projectiles was transported routinely, the order of projectiles supplied was adjusted by forward rotation and reverse rotation of the mechanism, when the transportation of projectiles was known and the location of the projectiles was known, the projectiles group was provided by the adjustment mechanism through similar distances. The CTP principle is shown in Fig. 4.

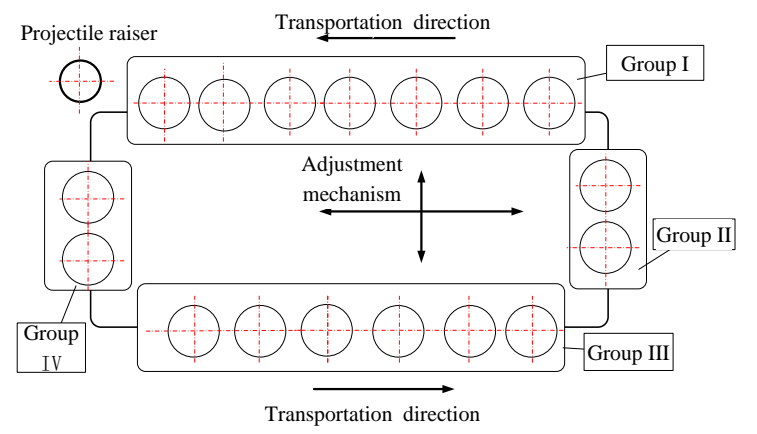

Fig. 4 Mechanism of platform-CTP

[Case Analysis] According to the operational requirements, use the clustering transportation projectiles method to transportation projectiles. In order to accomplish the target combat mission, it was assumed that each 10 projectiles transported as a group. Among them: the number and type of projectiles needed to be transported and those in transportation platform and storage tank are all random. The projectile transportation of the system was intelligently controlled by clustering ordering. The analysis results are shown in Tab.1 and tab. 2.

According to the cluster analysis formula (1), the grouping of random cluster projectile transportation is as follows: Group I(3,4,8,11,14,16), Group II(2,6,9,10,12,13), Group III(1,5,7).

Tab.1 Index and positon without CTP

\begin{tabular}{|c|c|c|c|c|c|c|c|c|c|c|c|c|c|c|c|c|c|}
\hline \multirow{2}{*}{\multicolumn{2}{|c|}{ index }} & 1 & 2 & 3 & 4 & 5 & 6 & 7 & 8 & 9 & 10 & 11 & 12 & 13 & 14 & 15 & 16 \\
\hline & & $\mathrm{C}$ & B & A & A & C & B & $C$ & A & B & B & A & B & B & A & B & A \\
\hline \begin{tabular}{|l|}
1 \\
\end{tabular} & $A$ & & & 1 & 1 & & & & 1 & & & 1 & & & 1 & & 1 \\
\hline 2 & A & & & 1 & 1 & & & & 1 & & & 1 & & & 1 & & 1 \\
\hline 3 & C & 1 & & & & 1 & & 1 & & & & & & & & & \\
\hline 4 & A & & & 1 & 1 & & & & 1 & & & 1 & & & 1 & & 1 \\
\hline 5 & A & & & 1 & 1 & & & & 1 & & & 1 & & & 1 & & 1 \\
\hline 6 & B & & 1 & & & & 1 & & & 1 & 1 & & 1 & 1 & & 1 & \\
\hline 7 & A & & & 1 & 1 & & & & 1 & & & 1 & & & 1 & & 1 \\
\hline 8 & B & & 1 & & & & 1 & & & 1 & 1 & & 1 & 1 & & 1 & \\
\hline 9 & C & 1 & & & & 1 & & 1 & & & & & & & & & \\
\hline 10 & B & & 1 & & & & 1 & & & 1 & 1 & & 1 & 1 & & 1 & \\
\hline
\end{tabular}

Tab.2 Index and positon with CTP

\begin{tabular}{|c|c|c|c|c|c|c|c|c|c|c|c|c|c|c|c|c|c|}
\hline \multirow{2}{*}{\multicolumn{2}{|c|}{ index }} & 3 & 4 & 8 & 11 & 14 & 16 & 2 & 6 & 9 & 10 & 12 & 13 & 15 & 1 & 5 & 7 \\
\hline & & A & A & A & A & A & A & B & B & B & B & B & B & B & C & C & C \\
\hline 1 & A & 1 & 1 & 1 & 1 & 1 & 1 & & & & & & & & & & \\
\hline 2 & A & 1 & 1 & 1 & 1 & 1 & 1 & & & & & & & & & & \\
\hline 4 & A & 1 & 1 & 1 & 1 & 1 & 1 & & & & & & & & & & \\
\hline 5 & A & 1 & 1 & 1 & 1 & 1 & 1 & & & & & & & & & & \\
\hline 7 & $A$ & 1 & 1 & 1 & 1 & 1 & 1 & & & & & & & & & & \\
\hline 6 & B & & & & & & & 1 & 1 & 1 & 1 & 1 & 1 & 1 & & & \\
\hline 8 & B & & & & & & & 1 & 1 & 1 & 1 & 1 & 1 & 1 & & & \\
\hline 10 & B & & & & & & & 1 & 1 & 1 & 1 & 1 & 1 & 1 & & & \\
\hline 3 & C & & & & & & & & & & & & & & 1 & 1 & 1 \\
\hline 9 & C & & & & & & & & & & & & & & 1 & 1 & 1 \\
\hline
\end{tabular}




\section{Model Establishment and Analysis}

\subsection{Model of CTP in Petri net}
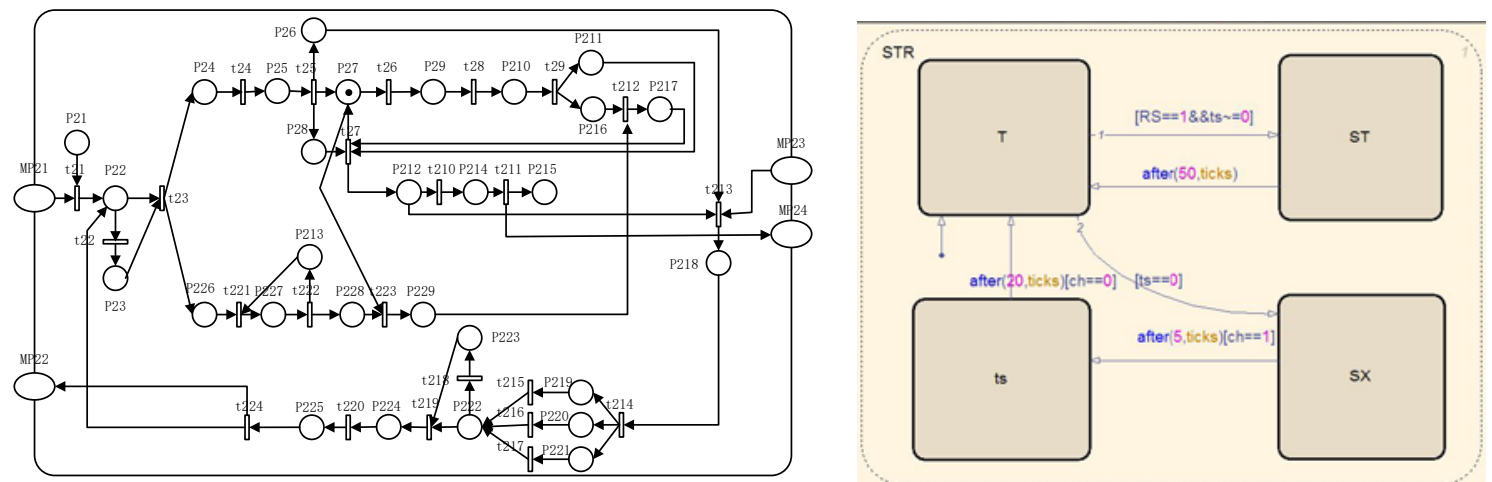

Fig.5 Random model of CTP in Petri net

Fig. 6 Dynamic logic relationship in Petri net

The model of CTP in Petri net was built as shown in Fig.5 and Fig.6. The meanings of each symbol are as follows: $\mathrm{T}$ is the positional module of platform projectiles; ST stands combination module of magazines and raise projectiles with control signal; SX is the switching module of different types of projectiles; ts is the selection module of platform projectiles. And rules are shown as follows:

Rule 1: The initial state assigns the information of the projectiles type in each location according to the random number generated. From No. 1 to No.16, the transfer position state of projectiles, are set to parallel state.

Rule 2: Platform steering signal is ts, if $\mathrm{TS}=0$, $\mathrm{SX}$ (projectile transportation type information assignment module) status activated.

Rule 3: Start-up signal of projectiles hoist is $\mathrm{RS}$. If $\mathrm{RS}=1$, and ts $\neq 0$, to activate $\mathrm{ST}$ (raising projectiles and supplementary projectiles) and start the raising work of projectiles.

Rule 4: After the SX state is executed, the state ts (platform turning judgement) is executed immediately; after the TS state is executed, the T (platform projectiles location operation) state is executed immediately.

Rule 5: The four states mentioned above are serial mechanisms, and no two states are activated at the same time during operation.

\subsection{Randommess analysis on real-time systems}
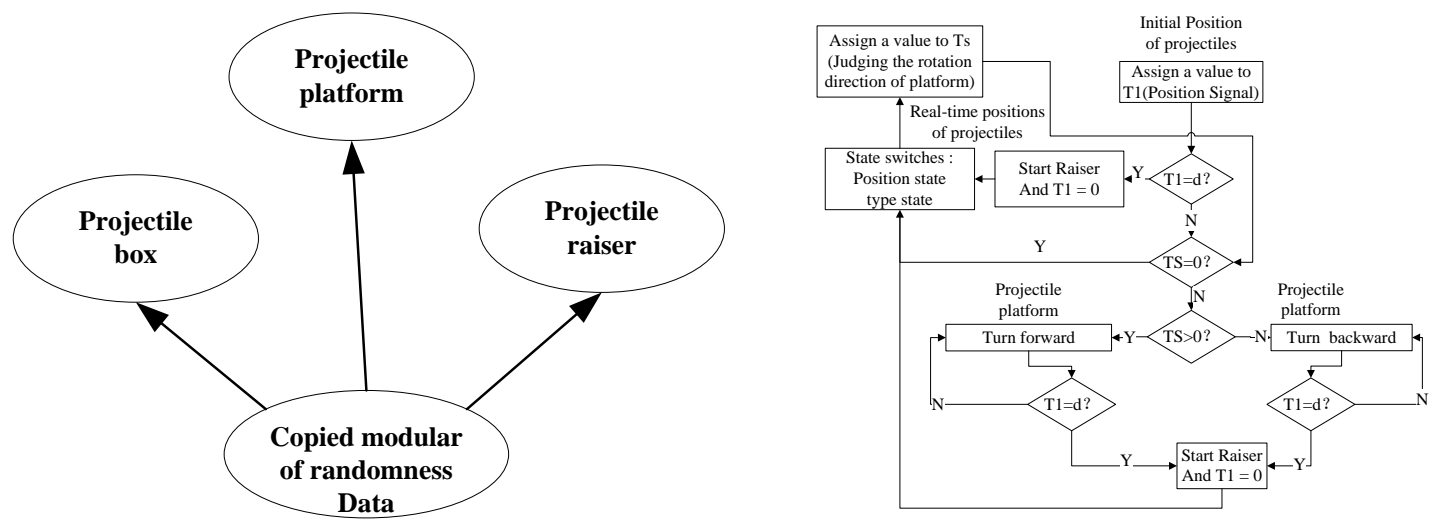

Fig.7 Decision model in Petri net

Fig. 8 The stochastic process of CTP in Petri net 
In order to ensure the randomness of sampling, Monte Carlo method was used for random sampling. The projectile transportation platform is in the 16 position. The quantity of projectiles in projectiles box is random, and the type is fixed. The horizontal axis is the position of projectiles, and the vertical axis is 1-A type projectiles, 2-B type projectiles and 3-C type projectiles respectively. The projectiles were grouped and the random number was automatically generated.

\section{Simulation and Validation}

The stochastic projectile transportation process controlled by Petri net is shown in Fig. 8. The logical state of the projectile transportation system was analyzed, and the sequential logic simulation analysis of projectiles transfer process was carried out by using Simulink and its sub-module in the state flow. Function simulation objectives are shown as follows:

1) To simulate the realization of the transit process of projectile transportation system;

2) To analyze the efficiency of the organization in the process of projectile transportation;

3) To determine the optimal scheduling path and timing of projectile transportation system.

Verify whether the designed system can transportation projectiles efficiently and intelligently under the circumstances that the position information of projectiles, the types of projectiles transported and the quantity of projectiles in the projectile magazines are random.

In the simulation test, the signal displayed by oscilloscope is the change curve of projectiles position information, the variety information curve of projectiles supplied randomly, and the change curve of projectiles quantity in projectile magazines. Random signals were random numbers generated by programs. The transportation efficiency of multi-types of projectiles is shown as Tab.3.

Tab.3 Real-time data of multiple types of projectiles

\begin{tabular}{|c|c|c|c|c|}
\hline Group & I & II & III & IV \\
\hline 1 & 32.83 & 30.83 & 38.83 & 35.83 \\
\hline 2 & 32.83 & 33.83 & 36.83 & 35.83 \\
\hline 3 & 29.83 & 29.83 & 30.83 & 29.83 \\
\hline 4 & 40.83 & 32.83 & 29.83 & 28.83 \\
\hline 5 & 30.83 & 35.83 & 35.83 & 40.83 \\
\hline 6 & 35.83 & 40.83 & 28.83 & 30.83 \\
\hline 7 & 50.83 & 38.83 & 27.83 & 30.83 \\
\hline 8 & 42.83 & 28.83 & 38.83 & 27.83 \\
\hline 9 & 35.83 & 27.83 & 26.83 & 38.83 \\
\hline 10 & 30.83 & 30.83 & 36.83 & 26.83 \\
\hline Total time & 363.3 & 330.3 & 331.3 & 326.3 \\
\hline
\end{tabular}

Four groups and 10 times each were simulated in its effectiveness of continuous test. And the results are shown in Tab.3. The average transportation rate of a group of projectiles (10 rounds) is $33.78 \mathrm{~s}$ and consistency evaluation index $\mathrm{C}$ is equal to 0.87 .

After 40 simulations, a total of 400 rounds of projectiles were supplied, including 148 rounds of a-type projectiles, 133 rounds of b-type projectiles and 119 rounds of c-type projectiles, which took totally 1351 seconds to transfer Projectile A. Therefore, it can be concluded that:

The average rates of projectiles were 9.13 s/round for Type A, 10.158 s/round for Type B and 11.35 s/round for Type C. According to the simulation curve, it can be concluded that the time required for a sequential projectile transportation cycle is $4.58 \mathrm{~s}$.

A projectile transportation cycle time of intercept stable operation stage is $2.15 \mathrm{~s}$, and its projectile 
transportation per minute is $27.91 \mathrm{~s}$, and the transportation efficiency of A-type projectiles in this transportation order is $55.81 \mathrm{rad} / \mathrm{min}$, that of B-type is $55.81 \mathrm{rad} / \mathrm{min}$ and of C-type is $27.91 \mathrm{rad} / \mathrm{min}$, which is consistent with the theoretical calculation results.

\section{Conclusions}

In summary, through comparing the simulation verification result of the two projectile transportation's reachability and efficiency, we get the following conclusion:

1) According to the simulation verification of the decision-making method of projectile transportation under the actual combat situation, the projectile transportation platform, which takes the method of CTP, realized transporting and replenishing projectiles continuously and efficiently with the conditions of compatible projectile transportation and replenishment.

2) Compared with the method of RTP, under normal circumstances, the space rate and ship-borne suitability of projectile transportation platform are significantly improved and the efficiency of projectile transportation is improved.

3) The CTP method in Petri net provides the necessary technical reserves for the development of new intelligent transportation of projectiles and compatible use of multiple types of weapon systems.

\section{References}

[1] ZHANG Ling-hai, ZHANG Yi-qun, LIU Zhi-wei. Current Situation and Development Trend of Autoloader in Chinese Medium and Large Caliber Naval Gun [J]. Computer Knowledge and Technology, 2016,12(27):256-258.

[2] BAI Yi, ZHOU Ke-qiang, DING Ji-liang. Architecture of Information-based Naval Gun Weapon System [J].Comma nd Control and Simulation,2017,39(01):20-24.

[3] Hsieh T Y, Chen T H, Yang N C, et al. Efficient network fault analysis method for unbalanced microgrid systems[J]. International Journal of Electrical Power \& Energy Systems, 2018, 103:89-101.

[4] Hai Feng Xu. The Research of Armored Vehicles Weapon System Fault Diagnosis Method [J]. Applied Mechanics and Materials,2014, 3561(678). 